\title{
Anabases
}

ANABASES Traditions et réceptions de l'Antiquité

$22 \mid 2015$

Varia

\section{Compléments au Mémorial Antoine Meillet}

Jean Loicq

\section{OpenEdition}

Journals

Édition électronique

URL : http://journals.openedition.org/anabases/5503

DOI : 10.4000/anabases.5503

ISSN : 2256-9421

\section{Éditeur}

E.R.A.S.M.E.

Édition imprimée

Date de publication : 20 octobre 2015

Pagination : 242-247

ISSN : 1774-4296

\section{Référence électronique}

Jean Loicq, « Compléments au Mémorial Antoine Meillet », Anabases [En ligne], 22 | 2015, mis en ligne le 20 octobre 2018, consulté le 21 octobre 2019. URL : http://journals.openedition.org/anabases/5503 ; DOI : 10.4000/anabases.5503 
à son maître Auguste Carrière) et que son travail suscite d'autres vocations pour explorer les fonds d'archives des savants français et étrangers susceptibles de contenir des lettres de Meillet ${ }^{35}$. C'est à ce prix que l'on pourra rendre à Meillet toute la place qu'il mérite dans l'historiographie de la première moitié du $\mathrm{xx}^{\mathrm{e}}$ siècle.

Pierre Ragot pierrehenri.ragot@wanadoo.fr

\section{Compléments au Mémorial Antoine Meillet}

\section{Jean LoicQ}

Les notes qui suivent visent à compléter la documentation réunie dans le Mémorial Antoine Meillet publié à l'occasion du centenaire de son entrée au Collège de France (Igo6), dans Studia Indoeuropaea. Revue de mythologie et de linguistique comparée, t. III (2006), et à part (Bucarest). La bibliographie propre d'A. Meillet qui figure dans ce Mémorial se voulait elle-même un complément de celle, déjà très imposante, qu'au lendemain de sa mort son successeur Ém. Benveniste avait hâtivement dressée en appendice au long article biographique de $\mathrm{J}$. Vendryes ${ }^{36}$, et qui s'est révélée très incomplète.

Sans doute, en effet, l'œuvre de Meillet (r866-ıg36) est essentiellement celle du linguiste qui a dominé sa discipline durant le premier tiers du $\mathrm{xx}^{\mathrm{e}}$ siècle, explorant toutes les branches de la famille indo-européenne, œuvre dont l'essentiel ne pouvait échapper à la vigilance de Benveniste. Mais la curiosité de Meillet et son action n'en ont pas moins porté sur des aspects des sciences de l'homme et de l'actualité où la science du linguiste et l'impartialité du savant lui conféraient une autorité incontestée.

On a pu voir ici-même par la recension de P. Ragot quel intérêt il portait à la culture arménienne: non seulement à sa langue, qu'il a un temps enseignée à l'École des Langues Orientales (auj. l'INALCO), mais aussi à sa littérature et à son art médiéval, avec une prédilection pour son architecture religieuse, dont il appréciait la pureté et la netteté de lignes. Il importe de rappeler aujourd'hui, en cette année d'un centenaire douloureux, que dès la fin de la Première Guerre, et surtout à la veille de la Conférence de Lausanne (I923), Meillet a mis avec persévérance son autorité au service de la cause arménienne. Avec son successeur à l'École des Langues, Frédéric Macler, il devait fonder dès I92o la Revue des études

35 Ce type de recherche reste toutefois aléatoire. Ainsi, bien qu'Antoine Meillet ait entretenu une correspondance suivie avec son collègue et ami Joseph Vendryes, le Fonds Vendryes ne contient aucune lettre de Meillet, d'après l'enquête préliminaire menée par P.-Y. Testenoire, “Les manuscrits de Joseph Vendryes : premier état des lieux", in Archives et manuscrits de linguistes, éd. V. Chepiga et E. Sofia, Louvain-laNeuve, Academia-L'Harmattan s.a., 2014, p. 96.. 
arméniennes, disparue avec la grande crise des années I93o, mais que des temps meilleurs ont heureusement fait renaître.

D'autre part, Meillet n'a pas été moins préoccupé, à la même époque, de l'avenir des nationalités qui, délivrées de l'oppression des grands empires disloqués, réclamaient leurs droits à l'indépendance. Parmi elles, essentiellement, les peuples slaves occidentaux. Il s'était, dès ses débuts, intéressé aux langues du groupe slave, encore peu cultivées alors en France, et y avait consacré sa thèse principale de doctorat ès lettres. Il leur réservait une place régulière dans son enseignement de l'École des Hautes Études. Durant la guerre, il a pris part à Paris, et notamment avec le futur président tchèque E. Beneš, à des réunions préparatoires aux grandes conférences de la Paix, apportant sa compétence dans le futur découpage, combien délicat, qui devait aboutir à l'État tchécoslovaque et à l'État polonais. À la fin de ıgı 8 et au début de ı9ı1, il remettait aux bureaux du congrès de Versailles des rapports sur la situation historique de l'Arménie et de la Pologne. Dès ıgı6, il donnait à la Sorbonne des conférences destinées à éclairer et à sensibiliser sur ces questions l'élite de l'opinion française. Il préludait ainsi à la création, avec l'historien Ernest Denis, d'un Institut et d'une Revue d'études slaves, l'un et l'autre bientôt centenaires aujourd'hui.

Ces multiples activités ont donné lieu à des publications, parfois hors commerce, ou encore occasionnelles et éphémères, dont les bibliothèques parisiennes les mieux pourvues (BnF comprise) ne possèdent pas la totalité, et dont il est permis de penser que Meillet lui-même n'a pas toujours reçu ou conservé un exemplaire ${ }^{37}$. C'est dans la pensée d'en conserver la mémoire qu'a été composé le Mémorial, dont il revenait à la Société de linguistique de Paris d'assurer la publication. Tel n'a pas été le cas ${ }^{38}$.

Le Mémorial recense en outre $\mathrm{I}^{\circ}$ les plus significatifs des comptes rendus qui ont paru dans des revues autres que le Bulletin de la Société de linguistique où, entre igo8 et Ig36, l'essentiel de la production linguistique (ou susceptible d'intéresser les linguistes) est passée en revue de manière critique, souvent constructive et en partie encore actuelle, et où il est aisé de la retrouver, auteur par auteur; $2^{\circ}$ un choix d'études parues depuis ig36 sur tel ou tel aspect de la personnalité et de l'œuvre publiée.

Mais l'œuvre de Meillet - surtout extra-linguistique - est si vaste et si dispersée, et elle continue, d'autre part, de susciter tant d'études et d'essais, qu'après une dizaine d'années le Mémorial de 2006 nécessite un complément. Ce dernier aurait dû trouver place dans les Studia Indoeuropaea. Mais cette publication n'a pas survécu à son fondateur, Dan Sluşanschi. Le signataire de ces lignes est reconnaissant à la revue Anabases de bien vouloir l'accueillir à l'occasion du compte rendu de P. Ragot, qui révèle un aspect attachant de la personnalité d'Antoine Meillet.

37 Il peut être utile de signaler ici aux chercheurs qu'un bon nombre d'entre elles, y compris celles qui ont préparé le Congrès de Versailles, celles du Comité parlementaire d'études, etc., sont conservées à la Bibliothèque de documentation contemporaine (Univ. de Nanterre), où je les ai commodément consultées vers 2005. - L'essentiel de la bibliothèque de Meillet est conservé au Collège de France et, pour la partie slave et orientale, à l'INALCO.

38 Un petit nombre de tirages à part, imprimé à frais d'auteur à Bucarest, sont disponibles au prix coûtant à mon adresse e-mail : < loicq-berger@skynet.be >. 
Les trois rubriques précitées du Mémorial sont représentées ici, avec le système de numérotation qui y a été adopté, et lui-même adapté à celui de Benveniste.

\section{L'œuvre. Ouvrages et Articles}

I9o6

76 a. Stat'i po slavjanovedenïu, II (V. I. L amanskis, éd., I9o6). - Articles de slavistique.

I9I6

227 c. L'unité slave.

La Nation tchèque (Paris), nº 4 (I5 juin I916), p. 5I-52. - Résumé d'une conférence à l'Institut d'études slaves, IgI6);

I9I7

229 f. Le paléoslave.

Ibidem, $\mathrm{n}^{\circ} 2 \mathrm{I}$ ( $\mathrm{I}^{\mathrm{er}}$ mars I9I7), p. 333-334. - Résumé d'une conférence (cf. 227 c).

I92I

304 a. [Note sur lat. augur, augustus].

Fait suite à l'art. d'A. Ernout de même titre, Mém. de la Soc. de linguist., 22 (I92I), p. 238, reproduit dans A. E., Philologica (Paris, I946), p. 70-7I.

I922

327 a. [Note sur le nominatif-accusatif des thèmes en - n- et l'origine du type gr. onomázō]. Fait suite à l'art. de N. Troubetzкoy, La forme slave du nom.-acc. sing. des thèmes en -n-, Mém. de la Soc. de linguist., 22 (I92I), p. 257.

I 925

397 f. [Sur l'intérêt, pour la civilisation de l'Occident, d'un rapprochement avec l'Orient]. Les Cahiers du mois, n 9-Io, p. 3o3. - Réponse à un questionnaire sur le thème Les appels de l'Orient.

I929

P. 62 p. L'étymologie de gr. érkhomai

Rev. des études grecques, 42 (I929), p. LIII-LIV.

2009

II. Caractères généraux des langues germaniques. Réimpression de l'édition de IgI7 (Cambridge Univ. Press).

522. Linguistique et anthropologie. Reproduit dans $7^{*}$ [cf. ci-après, III. Études], p. 265-269. 2014

3o. Gandon, F. (éd.), Meillet en Arménie. Journaux et correspondance (I8gi, Igo3). Avec la collaboration d'A.-M. Fryba-Reber (Limoges, Lambert-Lucas, 20I4). 


\section{L'œuvre. Comptes rendus critiques}

I 913

225 a. Gemoll, M., Die Indogermanen im alten Orient (Leipzig, I9II)

Rev. Germanique, 9 (ı913), p. 264.

225 b. SteYrer, J., Der Ursprung und das Wachstum der Sprache indogermanischer Europäer (I9I3)

Ibidem, p. 623.

I9I4

237 a. Wilke, G., Kulturbeziehungen zwischen Indien, Orient und Europa (Würzburg, IgI3)

Ibidem, Iо (I9I4), p. 5oI-5o2.

I920

289 a. GReEn, A., The dative of agency. A chapter of Indo-Europ. case syntax (New York, I9I3)

Ibidem, II(I920), p. I77.

I928

346. Walde, A. et Pokorny, J., Vergleichendes Wörterbuch der indogerm. Sprachen, II, I-3 (Berlin et Leipzig, I926-I927).

Ibidem, i9 (1928), p. 40.

I93o

358 a. ID., idem, II, 4 et I, I-2 (I927-I928).

Ibidem, 2I (I93o), p. 37-40.

358 b. Kieckers, E., Handbuch der vergleichenden gotischen Grammatik (Munich, I928)

Ibidem, p. 47-48.

I93I

36I $a$. WALDE-PoKorny (cf. 346), I, 5 (I930); III (I93i).

Ibidem, 22 (I93I), p. 53; 4I9.

\section{III. Études et témoignages}

1923

22 a*. Dauzat, A., Les doctrines linguistiques de M. Meillet

La Nature, $\mathrm{n}^{\circ} 2567$ (I6 juin I923), p. 373-374.

1972

345 a*. Снавот, G., La géographie appliquée à la Conférence de la Paix en rgig. Une séance franco-polonaise. - La pensée géographique française contemporaine. Mélanges André Meynier (Saint-Brieuc, I972), p. IOI-Io5. Position d'A. Meillet sur le problème de Gdansk (Dantzig).

1996

260 a*. Fodor, F., A. M. et les langues de l'Europe: une manifestation de l'imaginaire linguistique des linguistes. - Travaux de linguistes de l'Univ. d'Angers, 7 (I996), p. I3І-I40. 
1998

26ob. De Lamberterie, Ch., Méthode comparative et approche typologique. - La linguistique, 34/I (r998), spéc. p. 25-29. - Sur la phonologie des " dorsales » en indo-européen commun. - Voir aussi n 22Ia*.

2003

302 a*. Moret, S., A. Meillet et l'indépendance nationale. - P. SÉriot (éd.), Contributions suisses au XIIIe Congrès mondial des slavistes. Ljubljana, 2003 (Berne, 2003), p. I84-I98.

2005

35o a*. Topalli, K., Gjuha shqipe ne veprat e A. Mejes (“ la langue albanaise dans les travaux d'A. M.»). - Dijetarët francejë për gjuhën, letersinë dhe kulturën shqiptare («Savants français sur la langue, la littérature et la culture albanaise ») [Shkodër, Université, 2005], p. 85-88.

2006

246 c*. Сомтет, R. M., O klassifikacii slavjanskogo glagola v pervoj polovine XX veka: Pol' Buaje, Sergej Karcevskij i A. Meje («la classification du verbe slave dans la ${ }^{\text {re }}$ moitié du $\mathrm{xx}^{\mathrm{e}}$ siècle » : P. Boyer, S. Karcevski et A. M. »). - Voprosyjazykoznanija, 54 (2006), p. I02-I22.

246 d*. Savatovski, D. Meillet, historiographie du comparatisme. Histoire, épistémologie, langage, 28 (2006), p. 89-104.

2007

29I a*. LoicQ, J., A. Meillet, l'Europe et les études classiques. - Folia electronica classica (Louvain-la-Neuve, n I4, juillet-déc. 2007). Publ. en ligne: folia electronica@fltr.ucl.ac.be

348 b*. Swiggers, P., Les vues de Meillet et de Tesnière sur le rôle des élites dans l'élaboration des langues de culture. - Begioni, L. et Muller, Cl. (éd.), Problèmes de sémantique et de syntaxe. Hommage à André Rousseau (Lille, 2007), p. 47-60.

348 c*. Swiggers, P., A. Meillet. De la grammaire comparée à la sociologie du langage. - Anamnèse, 2 (2008), p. I/II-I54 (portrait).

2009

302 b*. Moret, S., Linguistique et nouvel ordre européen. Autour de la Grande Guerre. Velmezova, K. et, P. SÉriot (éd.), Discours sur les langues et rêves identitaires = Cahiers de l'ILSL (Lausanne), p. I29-I4I.

7*. Trois linguistes (trop) oubliés: A. Meillet, S. Lévi, F. Brunot = Anamnèse, 5 (2009). Voir ci-dessous Bert, Gandon, Muller, Rousseau, Sala, Swiggers.

305 b*. Muller, J.-Cl., Meillet, Lévi et Brunot, leurs maîtres communs et leur place dans le paysage scientifique européen. - Cf. 7 *, p. 9-18.

348 d*. Swiggers, P., A. Meillet et sa visée de la linguistique générale. - Cf. 7*, p. 2I-40.

323 a*. Sala, M. et Colceriu, St., A. Meillet, le maître de nos maîtres. - Cf. 7*, p. 4I-5o.

265 a* Gandon, F., A. Meillet, confident de Saussure anagrammatiste: un indo-européen paradoxal. - Cf. $7 *$, p. 5I-78.

3ı7 a*. Rousseau, A., A. Meillet et L. Duvau: histoire d'une rivalité. - Cf. 7*, p. 79-ı03 
232 a*. Bert, J.-F., Histoire des mots et sociologie du langage: Meillet, Cohen et Haudricourt. - Cf. 7*, p. Iо5-іI5.

3o2 c*. Morê, S., A. M. i Versal'skij dogovor [“ M. et le traité de Versailles »]-Naučnye čtenija 2007. Materialy conferencii Sankt-Petersburg (2009), p. 18-28.

2010

202 a*. Adrados, F., Bernabé, A. et Mendoza, J., Manual of I.-E. linguistics, I (Louvain et Paris), p. I03-ıo9; I22-ı28. - Confirme la doctrine d'A. M. sur le caractère binaire du système des occlusives dorsales en indo-européen.

302 d*. Moret, S., Linguistique et nationalisme dans l'entre-deux-guerres : Meillet et l'unité slave. - Arrive, M., etc. (éd.), De la grammaire à l'inconscient. Dans les traces de Damourette et Pichon (Limoges, 2010), p. I33-I40.

302 e*. Moret, S. A. Meillet and the Armenian genocide. - Publié en ligne http://hiphilangsci. net/20I5/o7/23/program-august-decembre-2015/

$2 \mathrm{OII}$

$302 \mathrm{f}^{*}$. Moret, S., A. $M$ et le futur des empires après la Première Guerre mondiale. Langages, $\mathrm{n}^{\circ} \mathrm{I} 82$ (juin 20II), p. II-24. - Théorie du langage et politique des linguistes. 302 g*. Moret, S., Le triomphe des langues « démocratiques »: A. M et l'Europe nouvelle. - Velmezova, E., Langue(s). Langage(s). Histoire(s). Cahiers de l'ILSL (Lausanne), 3I, p. I33-152.

2013

302 h*. Moret, S., Élever un rempart : A. $M$ et l'unité des langues slaves. - ELoy, J.-M., etc. (dir.), Langues collatérales en domaine slave. Carnets d'Atelier socio-linguistique, 7 , p. I5-3I.

302 i*. Monet, S., Emprunts et vigueur des langues et des nations chez $A$. $\boldsymbol{M}$ : des exemples arméniens et albanais. - Velmezova, E. (éd.), Histoire de la linguistique générale et slave. Cahiers de l'ILSL (Lausanne), 37, p. I/55-157.

2015

302 j*. Monet, S. Faire œuvre nationale : la slavistique française et la guerre de IgI4-IgI8. - Velmezova, E. et Dobritstn, A. (éd.), L'ordre du chaos - le chaos de l'ordre. Hommages à Leonid Heller (Berne, etc. Peter Lang, 20I0), p. 295-3i3.

Je remercie M. S. Moret (Université de Tartu) de m'avoir communiqué ces références.

Jean Loicq Université de Liège Avenue Nandrin, 24

B-4I3o Esneux loicq-berger@skynet.be 